\title{
Salt consumption and the risk of chronic diseases among Chinese adults in Ningbo city
}

\author{
Yi Lin', Qiuhong Mei ${ }^{2}$ Xujun Qian ${ }^{3}$ and Tianfeng $\mathrm{He}^{2^{*}}$
}

\begin{abstract}
Background: Chronic diseases have become one of essential public health concerns, leading causes of mortality in China. It is related to the changes in dietary pattern and dietary behavior. The objectives are to assess daily salt intake in Chinese people living in Ningbo and to examine its relationship with health outcomes.

Methods: Our study used data from health and nutrition survey in 2017. This study included 2811 adults aged 1879 years (48\% males) from urban and rural areas in Ningbo. A food frequency questionnaire together with demographic, physical and medical questionnaires was used to collect dietary intake, demographic, lifestyle and medical information. Ordinal logistic regression was used in the statistical analysis.

Results: The mean daily salt intake $(13.0 \mathrm{~g} /$ day) of the participants was higher than the Chinese dietary reference intake (DRl, $6 \mathrm{~g} / \mathrm{d}$ ), which was related to higher risk of pre-hypertension and hypertension. Stratified by gender, education and lifestyle factors, daily salt intake was only significant in the blood pressure category (male: $P=0.048$; less education: $P=0.003$; urban: $P=0.006$; no regular physical activity: $P=0.005$, no regular smoking: $P=0.006$ ). Ordinal logistic regression model shows that daily salt intake was significantly associated with higher odds of developing hypertension.

Conclusion: The daily salt intake of the majority of citizens living in Ningbo exceeded Chinese DRI and may increase the risk of hypertension. Moreover, public health intervention of salt restriction is necessarily needed for the prevention and control the ongoing epidemic of chronic diseases.
\end{abstract}

Keywords: Salt intake, Nutrition and health survey, Chronic diseases, Obesity, Diabetes, Hypertension, Chinese

\section{Introduction}

During past 3-4 decades, China has been facing an epidemiological shift in morbidity and mortality from under-nutrition related infectious diseases to overnutrition related non-communicable diseases $(\mathrm{NCD})$ due to rapid population ageing, urbanization and lifestyle changes [1]. National Health and Family Planning Commission of the People's Republic of China reported that the prevalence of obesity, hypertension and diabetes among Chinese adults at 18 years old and above in 2012 were $30.1 \%, 25.2 \%$ and $9.7 \%$, respectively [2]. In China, about $87 \%$ of all-cause mortality in 2012 and $77 \%$ of

\footnotetext{
*Correspondence: hetfnbcdc@163.com

${ }^{2}$ Department of Health and Education, Ningbo Municipal Center for Disease Control and Prevention, 237 Yongfeng Road, Ningbo 315010, China Full list of author information is available at the end of the article
}

total disability-adjusted life-year loss in 2010 were attributed to NCDs $[2,3]$.

Nutrition and dietary patterns have been the major modifiable determinants of chronic diseases [4-6]. Significant evidence has proved that human's diet has strong impact on health [5-8]. With increasing consumption of energy-dense foods in China, overconsumption of salt has been identified as a risk factor for development of major chronic diseases [9, 10], although salt/sodium not only is an important preservative method [11], but also makes essential contribution to eating pleasure and satisfaction [12]. Due to an important flavor, salt/sodium can determine the changes of dietary behavior, food choices and food preference [12]. Previous studies showed that high consumption of salt is positively associated with obesity having great body mass 
index (BMI) and waist circumference, hypertension, diabetes, cardiovascular diseases $[13,14]$.

For the past decades, salt intake has been increased gradually, exceeding the recommended daily intake $(5 \mathrm{~g} /$ d) proposed by world health organization (WHO) [15]. Results from the Chinese nutrition and health surveillance in 31 provinces with a 3-day food weighed record [16] and the China nutrition and transition cohort study covering 15 provinces using a standard three consecutive 24-h retrospective method [17] showed that the nationwide salt intake is $9.6 \mathrm{~g} / \mathrm{d}$ in $2010-2012$ and $9.9 \mathrm{~g} / \mathrm{d}$ (sodium: $3960.0 \mathrm{mg} / \mathrm{d}$ ) in 2015, respectively. Both studies reported that men consumed higher salt than women, middle aged group in particular [17]. A recent study showed that salt intake was $8.9 \mathrm{~g} / \mathrm{d}$ in 2015 in Zhejiang province [18].

Information on salt intake in Ningbo is not well studied and updated. Therefore, the aims of the current study are (1) to estimate the intake levels of salt in a representative sample of Ningbo, China and (2) to examine the association between salt intake and risk factors of health outcome including the status of BMI, fasting blood glucose and blood pressure.

\section{Methodology}

\section{Study design and study sample}

This current study is the baseline study of a local cohort survey conducted in Ningbo in 2017. A multistage, random cluster sampling procedure was used to draw the target samples, covering socio-economic status, public resources and health aspects in both urban and rural areas. A weighting method was carried out further based on the method of the sixth Ningbo census survey in 2010 from Ningbo Bureau of Statistics, following a national standardized sampling procedure proposed by $\mathrm{Na}-$ tional Bureau of Statistics of China [19]. The weighing sampling procedure considering the characteristics of urban-rural areas was to ensure a representativeness of socio-economic status. The urban and rural areas were categorized based on the definition proposed by the government of Ningbo. The counties of Cixi, Yuyao, Ninghai and Xiangshan are categorized into rural areas and the rest regions (Beilun, Fenghua, Jiangbei, Haishu, Yinzhou, and Zhenhai) are urban areas. City, town, county and village were stratified by income (low, middle, and high) in Ningbo. Four street blocks or counties were selected at each city/town level as primary sampling units. Two neighborhood committees or villages were selected as secondary sampling units at each city, town and county level. Approximately 120 out of 750 household families from each residential region were randomly selected in each neighborhood committee or village. KISH method, which is a way of randomly choosing target respondents for a household survey, was used to select one family member aged 15 to 80 years at the final sampling procedure, until 100 family members in each residential region were interviewed and recruited in this survey [20]. In total, 4973 individuals participated in the baseline study.

In the current study, 2963 out of 4973 recruited local citizens aged 18 to 79 years, who were not pregnant or lactating during data collection, not suffering from mental, physical diseases, severe chronic diseases, completing a food frequency questionnaire (FFQ) together with additional questionnaires including demography, lifestyle and self-health check report were included for the final data analysis.

The current study was conducted according to the guidelines proposed by Ningbo Municipal Center for Disease Control and Prevention (CDC) and all procedures involving human subjects were approved by Institutional Review Board of Ningbo Municipal CDC [No. IRB 201603]. Written and verbal informed consent was obtained from all participants.

\section{Dietary intake assessment}

A standard FFQ was developed based on the guidelines proposed by the China CDC and Ningbo CDC [21]. This FFQ was used to collect participants' quantitative dietary information on usual foods and dietary behavior. Dietary information was collected through 5 sections including family dietary background, family dietary behavior on seasoning, personal dietary behavior, alcohol consumption and water consumption. The section of family dietary background focused on how many family members usually had daily meals (breakfast, lunch and dinner) at home for the past month. Information of personal dietary behavior was on how often participants ate at home and outside for the past week. Personal dietary behavior for the past month was about the frequency of usual foods consumed (fresh vegetables, legumes and legume products, fresh fruit, red meat, poultry and shrimp, milk and milk products, eggs and egg products, barbeque and pickles). The section of family dietary behavior on seasoning focused on the information related to the amount of seasoning (salt, soya source, eatable oil and MSG) consumed for the past month. Dietary information of seasoning consumption was collected through 2 questions [How many packages/bottles did you eat for the past month? What was the quantity of one package/bottle for those consumed seasoning (g or litre)].The section of alcohol consumption was about when participants started drinking behavior, how many litres/ bottles and types of alcohol they drank for the past week. The section of water consumption was about whether participants drank water/tea/coffee, how much ( $200 \mathrm{ml}$ as the unit of a glass) and how often they drank for the past week. All the participants were interviewed 
by well-trained social healthcare workers from local health centers/hospitals and researchers from Ningbo CDC. The misreporting of dietary information has been correlated and excluded by experienced researchers during the field visit and statistical method. For example, salt intakes extremely higher than the daily reasonable consumption were excluded in the current study.

\section{Anthropometric measurements}

Weight $(\mathrm{kg})$ and height $(\mathrm{m})$ were self-reported to the $0.1 \mathrm{~kg}$ and the $0.1 \mathrm{~cm}$, respectively. Thereafter, BMI was calculated as weight $(\mathrm{kg}) /$ height $\left(\mathrm{m}^{2}\right)$. Participants were classified into four BMI categories according to China Obesity Task Force as follow: underweight $(<18.5 \mathrm{~kg} /$ $\left.\mathrm{m}^{2}\right)$, normal weight $\left(18.5-23.9 \mathrm{~kg} / \mathrm{m}^{2}\right)$, overweight $(24.0-$ $\left.27.9 \mathrm{~kg} / \mathrm{m}^{2}\right)$, and obesity $\left(\geq 28.0 \mathrm{~kg} / \mathrm{m}^{2}\right)[22]$.

\section{Biomarker measurements}

Biomarker information including blood pressure and fasting blood glucose was reported by healthcare workers at local health centers and hospitals. Blood pressure was measured at the upper limb brachial artery in a seated posture by an electronic blood pressure meter after participants were asked to rest for 5 mins. Blood pressure was measured 3 times at 30-second intervals. Therefore, the average blood pressure was calculated based on these 3 measurements. Participants, who were willing to be involved in the blood sampling, were asked to fast after $8 \mathrm{pm}$ on the previous day. In addition, medical questionnaire, including fasting status, acute infection, allergies, smoking, family medical history and medication, was completed by the participants for assessing blood pressure.

Systolic blood pressure (SBP) and diastolic blood pressure (DBP) were reported to the $0.1 \mathrm{mmHG}$ and blood glucose was to the $0.1 \mathrm{mmol} / \mathrm{L}$. Blood pressure and fasting blood glucose were measured following the instruction of Chinese national guidelines for the prevention and control of hypertension in primary care [23] and national guidelines for the prevention and control of type 2 diabetes in primary care [24], respectively.

Blood pressure was defined as 4 following categories: low blood pressure $(70 \leq \mathrm{SBP}<90$ and $40 \leq \mathrm{DBP}<80)$, normal blood pressure $(90 \leq \mathrm{SBP}<120$ and $60 \leq \mathrm{DBP}<$ $80)$, high blood pressure /pre-hypertension $(120 \leq \mathrm{SBP}<$ 140 and/or80 $\leq$ DBP < 90) and hypertension $(\geq 140)$ [25]. Fasting blood glucose was categorized into 3 groups: low level $(<4.0)$, normal level $(4.0-6.9)$ and high level/diabetes $(\geq 7.0)[24]$.

\section{Level of education}

Participants were asked to report the highest degree that they had obtained during the interview. Three categories of education level were created: (1) no education or lower secondary education; (2) secondary education; (3) higher education (bachelor, master or above).

\section{Statistical analysis}

The percentage and mean intake with standard error among risk groups were presented as descriptive analysis. Based on Central Limit Theorem, normal distribution was applied to daily salt intake across factor groups and health outcomes. Equality of the variances was examined using Levene's test. Statistical differences of the prevalence among the groups of dietary reference intake (DRI) and health outcomes were assessed using $\mathrm{Z}$ test. Student's T-test and ANOVA with Bonferroni correction/ Games-Howell were used to examine the difference of mean intakes within risk groups.

A two-tailed partial correlation was used to examine the correlation between daily salt intake and health outcomes (the status of BMI, fasting blood glucose and blood pressure), after adjusting for confounding factors (gender, age and education level) and lifestyle factors (regular smoking longer than 6 months, regular alcohol drinking longer than 6 months and regular physical activity). Ordinal logistic regression was fitted to investigate the association of salt intake (continuous variable) and health outcomes through three models:(1) unadjusted model; (2) model adjusted for gender, age and education level; (3) model further adjusted for lifestyle factors based on Model 2. Two-way interactions were examined between independent variable and confounding factors, and lifestyle factors in Model 3 as well. Interactions were only maintained in Model 3 if they were statistically significant. Statistical power was examined and higher than $80 \%$.

Results were considered statistically significant at a two-tailed level of 0.05 . Statistical analyses were conducted using the STATA statistical software package version 15 (2017).

\section{Results \\ Study population}

In total, 2811 (48\% men) out of 4973 recruited individuals participated in the survey and completed a FFQ with valid demographic, lifestyle and medical information. Among all 2963 individuals who completed the FFQ and other relevant questionnaires, 4 individuals had invalid BMI, 57 individuals got misreported fasting blood glucose, 48 individuals had extremely low/high SBP and 59 individuals had extremely low SBP.

Approximately $40 \%$ participants were living in rural areas. Around 21\% participants were in the group with higher education, young adult (18-40 years) in particular (Table 1). 87\% participants had medium monthly household income (6000-180,000/year). Almost half participants had regular physical activity, while the majority 
Table 1 Socio demographic characteristics of Chinese citizen living in Ningbo municipal center stratified by age

\begin{tabular}{|c|c|c|c|c|}
\hline \multirow[t]{2}{*}{ Socio demographic characteristic } & \multirow{2}{*}{$\begin{array}{l}\text { Total } \\
\text { (n) }\end{array}$} & \multicolumn{3}{|c|}{ Age (\%) } \\
\hline & & $18-40$ & $41-65$ & $>65$ \\
\hline \multicolumn{5}{|l|}{ Sex } \\
\hline Male & 1354 & 28 & 64 & 9 \\
\hline Female & 1457 & 30 & 61 & 9 \\
\hline \multicolumn{5}{|l|}{ Ethic group } \\
\hline Han & 2799 & 29 & 62 & 9 \\
\hline \multicolumn{5}{|l|}{ Geography } \\
\hline Urban & 1687 & 30 & 60 & 10 \\
\hline Rural & 1124 & 27 & 66 & 7 \\
\hline \multicolumn{5}{|l|}{ Education } \\
\hline No education or lower secondary & 1769 & 11 & 77 & 13 \\
\hline Secondary & 462 & 41 & 55 & 3 \\
\hline Higher education & 580 & 76 & 24 & 1 \\
\hline \multicolumn{5}{|l|}{ Occupation } \\
\hline Public service (e.g. teachers, administers at governments) & 356 & 58 & 40 & 2 \\
\hline Laborers (e.g. workers, famers) & 1260 & 16 & 73 & 11 \\
\hline Students & 38 & 92 & 8 & 0 \\
\hline Other sectors (e.g. business) & 482 & 46 & 51 & 3 \\
\hline Others & 675 & 22 & 66 & 12 \\
\hline \multicolumn{5}{|l|}{ Household Income } \\
\hline Low $(<6000 / y)$ & 24 & 17 & 58 & 25 \\
\hline Medium (6000-180,000/y) & 2441 & 26 & 64 & 9 \\
\hline $\operatorname{High}(\geq 180,000 / y)$ & 346 & 48 & 49 & 4 \\
\hline \multicolumn{5}{|l|}{ Regular Physical Activity } \\
\hline Yes & 1402 & 27 & 63 & 10 \\
\hline No & 1410 & 31 & 62 & 7 \\
\hline \multicolumn{5}{|l|}{ Regular Smoking } \\
\hline Yes & 611 & 22 & 70 & 8 \\
\hline No & 2200 & 31 & 60 & 9 \\
\hline \multicolumn{5}{|l|}{ Regular Alcohol Drinking } \\
\hline Yes & 570 & 16 & 75 & 10 \\
\hline No & 2241 & 32 & 59 & 8 \\
\hline \multicolumn{5}{|l|}{ BMI } \\
\hline Underweight & 205 & 60 & 34 & 6 \\
\hline Normal Weight & 1699 & 30 & 62 & 8 \\
\hline Overweight & 754 & 20 & 70 & 10 \\
\hline Obesity & 153 & 24 & 64 & 12 \\
\hline \multicolumn{5}{|l|}{ Fasting blood glucose } \\
\hline Low & 80 & 39 & 55 & 6 \\
\hline Normal & 2616 & 30 & 62 & 8 \\
\hline High/Diabetes & 115 & 7 & 77 & 17 \\
\hline \multicolumn{5}{|l|}{ Blood pressure } \\
\hline Low & 4 & 25 & 75 & 0 \\
\hline Normal & 417 & 53 & 45 & 3 \\
\hline Pre-hypertension & 2057 & 27 & 65 & 8 \\
\hline Hypertension & 333 & 10 & 71 & 20 \\
\hline
\end{tabular}


reported healthy lifestyles with no regular smoking (78\%) and no regular alcohol drinking (80\%). Considering health outcomes of chronic diseases, $26.8 \%$ and $5.4 \%$ participants were defined as overweight and obesity, respectively. Only $4 \%$ individuals were reported to have high fasting blood glucose status and tend to develop type 2 diabetes. On the contrary, 73\% individuals were considered to have pre-hypertension, the middle age ones (41-65 years, 65\%) in particular.

\section{Daily salt intake and risk factors among health outcomes}

The mean salt intake was $13.0 \mathrm{~g} / \mathrm{d}$ (median: $10 \mathrm{~g} / \mathrm{d}$ ) among all the participants. Men consumed almost the same daily salt intake as women did (men: $13.0 \mathrm{~g} / \mathrm{d}$; women: $12.9 \mathrm{~g} / \mathrm{d}$ ). Considering the age factor, senior generation (> 65 years, $14.4 \mathrm{~g} / \mathrm{d}$ ) consumed significantly more salt compared to young adults $(12.0 \mathrm{~g} / \mathrm{d})(P=$ $0.003)$. Additionally, rural participants $(13.6 \mathrm{~g} / \mathrm{d}) \mathrm{had}$ significantly more daily salt intake compared to urban ones $(12.5 \mathrm{~g} / \mathrm{d})(P=0.027)$.

Daily salt intake was much higher than Chinese RDI ( $\geq 6 \mathrm{~g} / \mathrm{d}) \quad(84 \%$ participants, $82 \%$ men and $85 \%$ women). Participants exceeding Chinese DRI were observed to have significantly higher prevalence of overweight/obesity, high fasting blood glucose status, prehypertension among all groups of gender, age and residential areas as compared to those counterparts (Table 2). Additionally, the prevalence of daily salt intake exceeding Chinese DRI $(\geq 6 \mathrm{~g} / \mathrm{d})$ was significantly higher than the prevalence of daily salt intake less

Table 2 Prevalence (\%) of health outcomes in different risk groups by Chinese recommended daily intake

\begin{tabular}{|c|c|c|c|c|c|c|c|}
\hline Dietary reference intake & $\begin{array}{l}\text { Normal } \\
\text { Weight }\end{array}$ & $\begin{array}{l}\text { Overweight/ } \\
\text { obesity }\end{array}$ & $\begin{array}{l}\text { Normal Fasting } \\
\text { Blood Glucose }\end{array}$ & $\begin{array}{l}\text { High Fasting Blood } \\
\text { Glucose/Diabetes }\end{array}$ & $\begin{array}{l}\text { Normal Blood } \\
\text { Pressure }\end{array}$ & Pre-hypertension & Hypertension \\
\hline \multicolumn{8}{|l|}{ Total } \\
\hline$<6 \mathrm{~g} / \mathrm{d}$ & 10 & $5^{b}$ & 15 & $1^{b}$ & 3 & $12^{d}$ & $1^{\text {fh }}$ \\
\hline$\geq 6 \mathrm{~g} / \mathrm{d}$ & $50^{j}$ & $24^{\mathrm{bj}}$ & $78^{j}$ & $3^{\mathrm{bj}}$ & $42^{j}$ & $61^{\mathrm{dj}}$ & $11^{\text {fhj }}$ \\
\hline \multicolumn{8}{|l|}{ Male } \\
\hline$<6 \mathrm{~g} / \mathrm{d}$ & 10 & $7^{b}$ & 16 & $1^{b}$ & 2 & $14^{d}$ & $2^{\text {fh }}$ \\
\hline$\geq 6 \mathrm{~g} / \mathrm{d}$ & $48^{j}$ & $30^{\mathrm{bj}}$ & $77^{j}$ & $3^{\mathrm{bj}}$ & $8^{j}$ & $36^{\mathrm{dj}}$ & $11^{\text {fhj }}$ \\
\hline \multicolumn{8}{|l|}{ Female } \\
\hline$<6 \mathrm{~g} / \mathrm{d}$ & 10 & $3^{b}$ & 14 & $0^{\mathrm{b}}$ & 4 & $10^{d}$ & $1^{\text {fh }}$ \\
\hline$\geq 6 \mathrm{~g} / \mathrm{d}$ & $53^{j}$ & $25^{\mathrm{bj}}$ & $79^{j}$ & $4^{\mathrm{bi}}$ & $15^{j}$ & $60^{\mathrm{dj}}$ & $10^{\mathrm{fhj}}$ \\
\hline \multicolumn{8}{|l|}{$18-40$} \\
\hline$<6 \mathrm{~g} / \mathrm{d}$ & 11 & $5^{b}$ & 18 & 0 & 5 & $13^{d}$ & $0^{e g}$ \\
\hline$\geq 6 \mathrm{~g} / \mathrm{d}$ & $52^{j}$ & $18^{\mathrm{bj}}$ & $78^{j}$ & $1^{\mathrm{bj}}$ & $22^{j}$ & $56^{\mathrm{dj}}$ & $4^{\text {th }}$ \\
\hline \multicolumn{8}{|l|}{$41-65$} \\
\hline$<6 \mathrm{~g} / \mathrm{d}$ & 10 & $5^{\mathrm{b}}$ & 14 & $1^{b}$ & 2 & $12^{d}$ & $1^{\text {th }}$ \\
\hline$\geq 6 \mathrm{~g} / \mathrm{d}$ & $50^{\mathrm{j}}$ & $31^{\mathrm{bj}}$ & $78^{j}$ & $0^{b}$ & $9^{j}$ & $64^{d j}$ & $12^{\mathrm{fhj}}$ \\
\hline \multicolumn{8}{|l|}{$>65$} \\
\hline$<6 \mathrm{~g} / \mathrm{d}$ & 9 & $5^{b}$ & 15 & $1^{a}$ & 1 & $12^{d}$ & $2^{\mathrm{fg}}$ \\
\hline$\geq 6 \mathrm{~g} / \mathrm{d}$ & $47^{j}$ & $33^{\mathrm{bj}}$ & $78^{j}$ & $7^{b}$ & 4 & $57^{\mathrm{dj}}$ & $24^{\mathrm{fhi}}$ \\
\hline \multicolumn{8}{|l|}{ Urban } \\
\hline$<6 \mathrm{~g} / \mathrm{d}$ & 13 & $6^{b}$ & 11 & $1^{b}$ & 4 & $16^{d}$ & $1^{\text {th }}$ \\
\hline$\geq 6 \mathrm{~g} / \mathrm{d}$ & $50^{j}$ & $25^{\mathrm{bj}}$ & $81^{j}$ & $4^{\mathrm{bj}}$ & $11^{j}$ & $60^{\mathrm{dj}}$ & $9^{\text {fhj }}$ \\
\hline \multicolumn{8}{|l|}{ Rural } \\
\hline$<6 \mathrm{~g} / \mathrm{d}$ & 5 & $4^{b}$ & 21 & $1^{b}$ & 2 & $7^{d}$ & $1^{\text {fh }}$ \\
\hline$\geq 6 \mathrm{~g} / \mathrm{d}$ & $53^{j}$ & $31^{\mathrm{bj}}$ & $73^{j}$ & $3^{\text {bi }}$ & $14^{j}$ & $63^{\mathrm{dj}}$ & $13^{\mathrm{fhj}}$ \\
\hline
\end{tabular}

${ }^{a}$ Significant difference across categories of weight and blood glucose, $P<0.05$

${ }^{b}$ Significant difference across categories of weight and blood glucose, $P \leq 0.001$

'Significant difference between normal blood pressure and pre-hypertension, $P<0.05$

${ }^{\mathrm{d}}$ Significant difference between normal blood pressure and pre-hypertension, $P \leq 0.001$

'Significant difference between normal blood pressure and hypertension, $P<0.05$

f Significant difference between normal blood pressure and hypertension, $P \leq 0.001$

${ }^{9}$ Significant difference between pre-hypertension and hypertension, $P<0.05$

'Significant difference between groups of Chinese dietary reference intake, $P<0.05$

${ }^{j}$ Significant difference between groups of Chinese dietary reference intake, $P \leq 0.001$ 
than Chinese DRI across all the groups of gender, age and residential areas.

Table 3 shows that daily salt intake was significantly higher with increasing blood pressure status among all the participants $(P=0.006)$. Stratified by confounding factors, increasing daily salt intake was significantly associated with blood pressure status men $(P=0.048)$, urban areas $(\mathrm{P}=0.006)$ and less educated group $(P=0.003)$. Daily salt intake among higher educated individuals deceased with increasing blood pressure status, although no significance was found. In terms of lifestyle factors, on one hand, higher daily salt intake was significantly associated with blood pressure status $(P=0005)$ among the group of no regular physical activity; on the other hand, higher daily salt intake was significantly associated with blood pressure status among healthy lifestyle including no regular smoking $(P=0.005)$ and alcohol drinking $(P=0.006)$.

\section{Association between daily salt intake and health outcomes among risk groups}

The results of partial correlation show that daily salt intake was only associated with SBP and DBP $(P=$ 0.043 and $P=0.008$, respectively) (Table 4 ). The

Table 3 Mean daily salt intake among risk groups by socio-demographic and lifestyle factors

\begin{tabular}{|c|c|c|c|c|c|c|c|c|c|c|}
\hline \multirow[t]{2}{*}{ Characteristics } & \multicolumn{2}{|l|}{$\mathrm{BMl}$} & \multicolumn{2}{|c|}{$\begin{array}{l}\text { Fasting Blood } \\
\text { Glucose }\end{array}$} & \multicolumn{3}{|c|}{ Blood Pressure } & \multicolumn{3}{|c|}{$P$-value } \\
\hline & Normal & $\begin{array}{l}\text { Overweight/ } \\
\text { obesity }\end{array}$ & Normal & Diabetes & Normal & $\begin{array}{l}\text { Pre- } \\
\text { hypertension }\end{array}$ & Hypertension & $\mathrm{BMI}$ & $\begin{array}{l}\text { Blood } \\
\text { Glucose }\end{array}$ & $\begin{array}{l}\text { Blood } \\
\text { Pressure }\end{array}$ \\
\hline Total & $\begin{array}{l}13.0 \\
(0.330)\end{array}$ & $12.9(0.386)$ & $\begin{array}{l}13.0 \\
(0.254)\end{array}$ & $\begin{array}{l}13.3 \\
(1.2)\end{array}$ & $\begin{array}{l}12.4 \\
(0.538)\end{array}$ & $12.9(0.265)$ & $15.1(1.0)$ & 0.879 & 0.880 & 0.006 \\
\hline \multicolumn{11}{|l|}{ Sex } \\
\hline Male & $\begin{array}{l}13.3 \\
(0.537)\end{array}$ & $12.4(0.505)$ & $\begin{array}{l}13.0 \\
(0.395)\end{array}$ & $\begin{array}{l}13.3 \\
(1.5)\end{array}$ & $12.6(1.1)$ & $12.7(0.381)$ & $15.4(1.6)$ & 0.276 & 0.975 & 0.048 \\
\hline Female & $\begin{array}{l}12.7 \\
(0.403)\end{array}$ & $13.5(0.594)$ & $\begin{array}{l}12.9 \\
(0.324)\end{array}$ & $\begin{array}{l}13.3 \\
(1.7)\end{array}$ & $\begin{array}{l}12.3 \\
(0.600)\end{array}$ & $12.8(0.369)$ & $14.7(1.2)$ & 0.291 & 0.845 & 0.129 \\
\hline \multicolumn{11}{|l|}{ Geography } \\
\hline Urban & $\begin{array}{l}12.6 \\
(0.463)\end{array}$ & $12.4(0.556)$ & $\begin{array}{l}12.7 \\
(0.246)\end{array}$ & $\begin{array}{l}14.4 \\
(1.7)\end{array}$ & $\begin{array}{l}11.5 \\
(0.735)\end{array}$ & $12.3(0.359)$ & $15.7(1.8)$ & 0.788 & 0.259 & 0.006 \\
\hline Rural & $\begin{array}{l}13.6 \\
(0.433)\end{array}$ & $13.6(0.511)$ & $\begin{array}{l}13.3 \\
(0.515)\end{array}$ & $\begin{array}{l}11.4 \\
(2.4)\end{array}$ & $\begin{array}{l}13.8 \\
(0.763)^{a}\end{array}$ & $13.5(0.380)^{\mathrm{a}}$ & $14.4(0.894)$ & 0.959 & 0.486 & 0.569 \\
\hline \multicolumn{11}{|l|}{ Education } \\
\hline $\begin{array}{l}\text { No education or lower } \\
\text { secondary }\end{array}$ & $\begin{array}{l}13.5 \\
(0.465)\end{array}$ & $13.5(0.464)$ & $\begin{array}{l}13.5 \\
(0.346)\end{array}$ & $\begin{array}{l}13.9 \\
(1.4)\end{array}$ & $\begin{array}{l}11.9 \\
(0.590)\end{array}$ & $13.3(0.351)$ & $16.0(1.3)$ & 0.905 & 0.883 & 0.003 \\
\hline Secondary & $\begin{array}{l}12.7 \\
(0.746)\end{array}$ & $12.2(1.1)$ & $\begin{array}{l}12.6 \\
(0.611)\end{array}$ & $\begin{array}{l}11.4 \\
(1.6)\end{array}$ & $13.9(1.8)$ & $12.4(0.650)$ & $12.4(1.6)$ & 0.735 & 0.779 & 0.628 \\
\hline Higher education & $\begin{array}{l}11.6 \\
(0.501)\end{array}$ & $10.8(0.826)^{\mathrm{a}}$ & $\begin{array}{l}11.5 \\
(0.412)\end{array}$ & $9.4(3.2)^{\mathrm{a}}$ & $\begin{array}{l}12.2 \\
(0.893)\end{array}$ & $11.3(0.461)^{\mathrm{a}}$ & $9.2(1.1)^{b}$ & 0.414 & 0.487 & 0.264 \\
\hline \multicolumn{11}{|l|}{ Regular Physical Activity } \\
\hline Yes & $\begin{array}{l}12.2 \\
(0.400)\end{array}$ & $12.1(0.513)$ & $\begin{array}{l}12.4 \\
(0.322)\end{array}$ & $\begin{array}{l}11.6 \\
(0.805)\end{array}$ & $\begin{array}{l}12.4 \\
(0.790)\end{array}$ & $12.1(0.343)$ & $13.5(0.993)$ & 0.860 & 0.592 & 0.288 \\
\hline No & $\begin{array}{l}13.7 \\
(0.524)^{\mathrm{a}}\end{array}$ & $13.7(0.575)^{\mathrm{a}}$ & $\begin{array}{l}13.6 \\
(0.392)^{\mathrm{a}}\end{array}$ & $\begin{array}{l}15.6 \\
(2.5)\end{array}$ & $\begin{array}{l}12.4 \\
(0.736)^{a}\end{array}$ & $13.4(0.402)^{\mathrm{a}}$ & $17.2(2.0)$ & 0.971 & 0.402 & 0.005 \\
\hline \multicolumn{11}{|l|}{ Regular Smoking } \\
\hline Yes & $\begin{array}{l}13.9 \\
(0.632)\end{array}$ & $12.5(0.676)$ & $\begin{array}{l}13.4 \\
(0.473)\end{array}$ & $\begin{array}{l}11.7 \\
(1.4)\end{array}$ & $12.5(1.3)$ & $13.3(0.528)$ & $13.9(1.1)$ & 0.140 & 0.442 & 0.794 \\
\hline No & $\begin{array}{l}12.7 \\
(0.382)\end{array}$ & $13.0(0.464)$ & $\begin{array}{l}12.9 \\
(0.287)\end{array}$ & $\begin{array}{l}13.8 \\
(1.5)\end{array}$ & $\begin{array}{l}12.4 \\
(0.585)\end{array}$ & $12.6(0.306)$ & $15.5(1.3)$ & 0.638 & 0.597 & 0.005 \\
\hline \multicolumn{11}{|l|}{ Regular Alcohol Drinking } \\
\hline Yes & $\begin{array}{l}14.0 \\
(0.651)\end{array}$ & $13.0(0.695)$ & $\begin{array}{l}13.5 \\
(0.488)\end{array}$ & $\begin{array}{l}12.0 \\
(1.7)\end{array}$ & $12.1(1.3)$ & $13.5(0.551)$ & $14.1(1.1)$ & 0.297 & 0.446 & 0.623 \\
\hline No & $\begin{array}{l}12.7 \\
(0.379)\end{array}$ & $12.9(0.457)$ & $\begin{array}{l}12 . \\
8(0.293)\end{array}$ & $\begin{array}{l}13.7 \\
(1.3)\end{array}$ & $\begin{array}{l}12.4 \\
(0.583)\end{array}$ & $12.6(0.302)$ & $15.4(1.3)$ & 0.816 & 0.633 & 0.006 \\
\hline
\end{tabular}


Table 4 Partial correlation of the relationship between daily salt intake and health outcomes at baseline study

\begin{tabular}{lll}
\hline Health Outcomes $^{\mathrm{a}}$ & \multicolumn{2}{l}{ Daily salt intake } \\
\cline { 2 - 3 } & $\mathrm{r}$ & $P$-value \\
\hline BMI & -0.009 & 0.642 \\
Fasting Blood Glucose & -0.008 & 0.680 \\
Systolic blood pressure & 0.038 & 0.043 \\
Diastolic blood pressure & 0.050 & 0.008 \\
\hline
\end{tabular}

${ }^{a}$ Controlling for gender, age, education, geography, physical activity, smoking and drinking

associations between health outcomes and daily salt intake were further assessed using ordinal logistic regression (Table 5). Significant associations between daily salt intake and blood pressure status were found in Model 1 $(P=0.014)$ and Model $3(P=0.044)$ after controlling for gender, age, education and lifestyle factors. No significant association was observed in Model 3. Interactions were not significantly in the models and removed from Model 3. The result in Model 3 indicates that increasing $1 \mathrm{~g} / \mathrm{d}$ daily salt intake would result in a 1.006 times odds of getting higher blood pressure status, after adjusting for the potential confounding factors and lifestyle factors.

\section{Discussion}

This large-scale population-based study is one of the first studies to examine the associations between daily salt intake and health outcomes including obesity, diabetes and hypertension among adult population in Ningbo, China.

The current study indicates that mean daily salt intake among adults was extremely higher than Chinese DRI $(6 \mathrm{~g} / \mathrm{d})$ [26] and WHO recommendation $(5 \mathrm{~g} / \mathrm{d})$ [15]. The majority of our participants (84\%) consumed more than the Chinese DRI level. Observational studies showed that the majority of Chinese adults had high salt consumption $[17,27,28]$, which is in line with our findings. One recent report from China Health and Nutrition Survey in 2002 showed that Chinese national level

Table $\mathbf{5}$ Ordinal regression analysis of the association between the status of blood pressure and daily salt intake among Chinese participants living in Ningbo city

\begin{tabular}{lllll}
\hline $\begin{array}{l}\text { Salt } \\
\text { intake }\end{array}$ & OR $^{\mathrm{c}}$ & $\begin{array}{l}\mathrm{P} \text { - } \\
\text { value }\end{array}$ & & $95 \% \mathrm{Cl}$ \\
\cline { 5 - 5 } & & & Lower Bound & Upper Bound \\
\hline Model 1 $^{\mathrm{b}}$ & 1.009 & 0.014 & 1.002 & 1.016 \\
Model 2 $^{\mathrm{b}}$ & 1.006 & 0.064 & 1.000 & 1.012 \\
Model 3 $^{\mathrm{b}}$ & 1.006 & 0.044 & 1.000 & 1.013
\end{tabular}

OR odds ratio; Cl: confidence interval

${ }^{a}$ Hypertension reference category in the model

${ }^{b}$ Model 1: Unadjusted; Model 2, adjusted for gender, age and education level; Model 3, Model 2 further adjusted for lifestyle factors of salt intake was $12 \mathrm{~g} / \mathrm{d}$, but the intake levels were diverse according to geography [29]. Our finding is slightly higher than the national level in 2002 as dietary behavior, food preparation and seafood in local area contributed to high amount of daily salt intake. Likewise, high salt intakes among adults were reported $18.0 \mathrm{~g} / \mathrm{d}$ in Turkey measured with 24-h urine collections [30]; 10.9 $\mathrm{g} / \mathrm{d}$ in India estimated with a three-day food diary [31]; $10.7 \mathrm{~g} / \mathrm{d}$ in Portugal using 24-h urine collections [32]; $9.2 \mathrm{~g} / \mathrm{d}$ in the USA with 24-h urine collections [33]; 11.5 $\mathrm{g} / \mathrm{d}$ in Italy with FFQ [34] and $9.0 \mathrm{~g} / \mathrm{d}$ in Australia using 24-h urine collections [35], although dietary assessments were different for collecting dietary information.

Our study showed that daily salt intake was the most strongly associated with higher odds of developing blood pressure status, which is consistent with several previous observational studies [36-38]. In contrast with blood pressure, obesity and fasting blood glucose were not significantly associated with daily salt intake. Results from the INTERMAP study in 1996-1999 involving 17 population samples aged 40-59 years showed that significantly adverse association of 24-h urinary dietary sodium excretion with blood pressure after with or without control for BMI [39]. Similarly, ALTURK study showed that salt intake together with gender, age and BMI was the significant predictors of both SBP and DBP [30]. The results from a worldwide epidemiologic studyINTERSALT study, conducted on dietary salt/sodium and hypertension with a 24-h urinary sodium, demonstrated the increased salt intake elevating blood pressure level with age [40]. The increase in salt sensitivity with age may result in a raise in salt intake. We found that senior generation had significantly higher daily salt intake compared to young adults in our study. Additionally, blood pressure of senior generation in the prehypertension and hypertension groups is more sensitive to daily salt intake [41, 42], although no significant difference in daily salt intakes was observed among obesity and diabetes status. Salt sensitivity could be genetic predisposition for Southeast Asian populations compared to Caucasians [43]. Therefore, senior Chinese generation is prone to develop prehypertension and hypertension due to salt sensitivity.

One conclusion from an epidemiological study indicated that processed foods as a strong risk factor for cardiovascular disease may cause an association of dietary salt with weight gain and obesity [41]. Excessive processed foods and dietary salt are related to passive overconsumption of energy, fat, cholesterol and fluid; may hereby contribute to weight gain [44]. Higher salt and energy intakes were reported among obese subjects [28], which is in contrast to our findings. No significant difference in daily salt intake was found among the status of BMI and fasting blood glucose. A supporting result 
from a Korean national health and nutrition examination survey indicated no association of sodium excretion with obesity and metabolic disorder in senior generation [41]. The possible reason is that those participants, who are categorized into obesity and diabetes groups, might be more careful about their dietary intake since they might have knowledge of weight gain, blood glucose, diabetes, and dietary instruction. A recent report from the UK National Diet and Nutrition Survey 2008/2009 to 2011/ 2012 indicated that a more $1-\mathrm{g} / \mathrm{d}$ salt intake was associated with $26 \%$ higher odds of overweight and obesity after adjusting the confounding factors, sociodemographic factors and total energy intake [45]. A high-salt diet may increase fasting ghrelin levels, thus, regulate appetite, glucose homeostatic, insulin resistance and fat accumulation [46], which most likely involves in the mechanism of obesity and diabetes. In addition, a high-salt diet rich in dietary $\mathrm{Na}^{+}$density causes dietary $\mathrm{K}^{+}$depletion, thus, results in negative impact on blood pressure regulation, and health benefits on cardiovascular diseases since high $\mathrm{Na}^{+}$density may increase left ventricle wall and mass [47].

Moreover, poor nutrition and sedentary lifestyle are the best-known inducers developing chronic diseases. Besides nutrition and lifestyle, education is an essential factor as well causing chronic diseases. Knowledge and awareness related to social class are the factors of determination of dietary behavior and lifestyle [28, 48]. A high dietary sodium may be a reflection of poor diet quality and energy-dense diet. Interestingly, women consuming more than Chinese DRI was found to have almost 2 times higher prevalence of pre-hypertension than men did in our study. One recent study indicated that rural Chinese women had higher incidence of hypertension compared to urban ones due to less physical activity and health education [49]. A healthy dietary pattern high in fruit, vegetables and low fat diet led to lower blood pressure compared to a high salt and energy-dense dietary pattern [50]. Although overall daily salt intake was higher among rural participants in our study, daily salt intake of urban participants significantly increased among blood pressure status, hypertension group in particular. The dietary pattern and culture in the rural areas are still keeping a traditional Chinese way, while the dietary pattern in the urban areas is more diverse and closer to the Western dietary pattern such as fast foods and semi-prepared foods. This energy-dense dietary pattern changing the traditional Chinese dietary pattern and dietary culture has caused higher blood pressure, reported from a national level study in China [51]. Evidence proved that the majority of individuals are lack of knowledge of DRI for salt, and its relationship of salt with hypertension, less educated individuals in particular [28]. Less education and lack of knowledge resulted in participants consuming excessive daily salt intake, which is significantly associated with higher odds of hypertension.

\section{Strengths and limitations}

The current study was the baseline study of a cohort survey, which was representative for the local Chinese citizens living in Ningbo, China. Additionally, it is the first study examining daily salt intake in relation to chronic diseases including obesity, diabetes and blood pressure in Ningbo. Biomarkers were reported by welltrained health workers.

Nonetheless, some limitations of this study need to be considered. First, causality cannot be inferred according to cross-sectional study design. Second, FFQ is not an accurate assessment collecting infrequently consumed foods. Then, total energy intake was not adjusted in the ordinal logistic regression model. Thus, it may influence associations of daily salt intake with health outcomes of chronic diseases. Moreover, information on dietary information relies on individuals' memory and might be biased towards misreporting.

\section{Conclusion}

Mean daily salt intake among the majority of participants exceeded Chinese DRI. Potential confounding and lifestyle factors were playing important roles in daily salt intake among risk groups, blood pressure in particular. Less educated and rural individuals, who were overweight/obese or had high fasting blood glucose/blood pressure, consumed significantly more salt than those counterparts did. Daily salt intake was significantly associated with higher odds of developing prehypertension and hypertension. Therefore, salt registration initiative is essential in the management, policy and strategy of salt content for preventing and controlling the epidemic prehypertension, hypertension and its related chronic diseases. The future study needs to investigate causality of prospective cohort study.

\section{Abbreviations \\ BMI: Body mass index; CDC: Center for Disease Control and Prevention; Cl: Confidence interval; DBP: Diastolic blood pressure; DRI: Dietary reference intake; FFQ: Food frequency questionnaire; NCD: Non-communicable diseases; OR: Odds ratio; SBP: Systolic blood pressure; WHO: World health organization}

\section{Acknowledgements}

We thank all the participants involved in the survey. We thank Ningbo Municipal CDC for their support of the data collection. We thank Prof. Zhuo Chen from Center for Health Economics, School of Economics and Prof.

Stephen Morgan from Nottingham China Health Institute for their support of research collaboration between University of Nottingham, Ningbo China and Ningbo Municipal CDC.

Availability of supporting data Not applicable. 


\section{Authors' contribution}

Lin Y performed and interpreted statistical analysis and drafted manuscript writing. He TF and Qian XJ contributed to the study design, reviewed and organized the field work. Mei QH was responsible for the field work, data collection and quality control. All authors read and approved the final manuscript.

\section{Funding}

This work was supported by Ningbo Science \& Technology Bureau [No. 2019A610391], the Medical Technology Program Foundation of Zhejiang, China [No. 2018KY730; No.2018KY680] and the Project of Zhejiang Public Welfare Fund [No. LGF19H260010].

\section{Ethics approval and consent to participate}

This study was conducted according to the guidelines proposed by Ningbo Municipal Center for Disease Control and Prevention and all procedures involving human subjects were approved by Institutional Review Board of Ningbo Municipal CDC [No. IRB 201603]. Written and verbal informed consent was obtained from all participants.

\section{Consent for publication}

Not applicable.

\section{Competing interests}

All authors have read and approved the final manuscript. All authors declare no conflicts of interest.

\section{Author details}

${ }^{1}$ Center for Health Economics, School of Economics, Faculty of Humanities and Social Sciences, University of Nottingham, Ningbo China, 199 Taikang East Road, University Park, Ningbo 315100, China. ${ }^{2}$ Department of Health and Education, Ningbo Municipal Center for Disease Control and Prevention, 237 Yongfeng Road, Ningbo 315010, China. ${ }^{3}$ Departmentof Health and Management, Ningbo First Hospital, 59 Liuting Street, Ningbo 315010, China.

Received: 25 September 2019 Accepted: 7 January 2020 Published online: 29 January 2020

\section{References}

1. Yu D, Shi J, Zhang H, Wang Z, Lu Y, Zhang B, Pan Y, Wang B, Sun P. Identifying patterns of non-communicable diseases in developed eastern coastal China: a longitudinal study of electronic health records from 12 public hospitals. BMJ Open. 2017;7:e016007.

2. National Health and Family Planning Commission of the People's Republic of China: 2014 Report on Chinese resident's chronic disease and nutrition. 2015.

3. World Health Organization. In: country profiles. Non communicable diseases progress monitor 2015. 2015; http://apps.who.int/iris/bitstream/10665/1846 88/1/9789241509459_eng.pdf. Accessed 15 May 2019.

4. Zhao $Y$, Wang $L$, Xue $H$, Wang $H$, Wang $Y$. Fast food consumption and its associations with obesity and hypertension among children: results from the baseline data of the childhood obesity study in China mega-cities. BMC Public Health. 2017;17:933.

5. Eggersdorfer M, Wyss A. Carotenoids in human nutrition and health. Arch Biochem Biophys. 2018;652:18-26.

6. Micha R, Penalvo JL, Cudhea F, Imamura F, Rehm CD, Mozaffarian D. Association between dietary factors and mortality from heart disease, stroke, and type 2 diabetes in the United States. Jama. 2017:317:912-24.

7. Slavin JL, Lloyd B. Health benefits of fruits and vegetables. Adv Nutr. 2012;3: 506-16.

8. Jhee JH, Kee YK, Park JT, Chang TI, Kang EW, Yoo TH, Kang SW, Han SH. A diet rich in vegetables and fruit and incident CKD: a community-based prospective cohort study. Am J Kidney Dis. 2019

9. Asaria P, Chisholm D, Mathers C, Ezzati M, Beaglehole R. Chronic disease prevention: health effects and financial costs of strategies to reduce salt intake and control tobacco use. Lancet. 2007;370:2044-53.

10. Nishida C, Uauy R, Kumanyika S, Shetty P. The joint WHO/FAO expert consultation on diet, nutrition and the prevention of chronic diseases: process, product and policy implications. Public Health Nutr. 2004;7(1A): $245-50$
11. Harlina PW, Ma M, Shahzad R, Gouda MM, Qiu N. Effect of clove extract on lipid oxidation, antioxidant activity, volatile compounds and fatty acid composition of salted duck eggs. J Food Sci Technol. 2018;55:4719-34.

12. Duffy VB, Hayes JE, Sullivan BS, Faghri P. Surveying food and beverage liking: a tool for epidemiological studies to connect chemosensation with health outcomes. Ann N Y Acad Sci. 2009:1170:558-68.

13. Meneton $P$, Jeunemaitre $X$, de Wardener HE, MacGregor GA. Links between dietary salt intake, renal salt handling, blood pressure, and cardiovascular diseases. Physiol Rev. 2005;85:679-715.

14. Moosavian SP, Haghighatdoost F, Surkan PJ, Azadbakht L. Salt and obesity: a systematic review and meta-analysis of observational studies. Int J Food Sci Nutr. 2017;68:265-77.

15. World Health Organization. In: Summary of evidence. Guideline: Sodium intake for adults and children. Geneva: World Health Organization; 2012. https://www.who.int/nutrition/publications/guidelines/sodium intake printversion.pdf. Accessed 15 May 2019

16. Yu D, He Y, Fang $H$, Xu X, Wang $X, Y u$ W, Jia F, Yang $X$, Ma G, Zhao L. Salt intake among Chinese adults in 2010-2012. Zhonghua Yu Fang Yi Xue Za Zhi. 2016:50:217-20.

17. Zhang JG, Wang ZH, Du WW, Su C, Jiang HR, Huang FF, Jia XF, Ouyang YF, Li L, Wang $Y$, et al. Dietary sodium intake of adult residents in 15 provinces of China in 2015. Zhonghua Yu Fang Yi Xue Za Zhi. 2019;53:455-8.

18. Qi X, Wang Z, Jiang M, Cheng G. Investigation on salt intake of residents in Sanmen county. Zhejiang J Prev Med. 2015;03:281-2.

19. National Bureau of Statistics of China: Regulation of Survey of Demographic Shift. 2018.

20. Cao Y, Chen J, Cao JW, Qian JC. Application of Kish grid sampling in world Health survey (China survey). Fudan University Press Med. 2004:31:307-10.

21. Kong F, Li H, Xu G, Ying Y, Gong Q, Zhao J, Zhang X, Zhang L, Liu S, Han L. Association of Dietary Behaviors and Sleep Quality: results from the adults chronic diseases and risk factors survey of 2015 in Ningbo, China. Int J Environ Res Public Health. 2018;15.

22. Department of Diseases Control and Ministry of Health of the People's Republic of China. The guideline of prevention and control of overweight and obesity among Chinese adults. Acta Nutrimenta Sinica. 2004:26.

23. National Cardiovascular Center. National guidelines for the prevention and control of hypertension in primary care (2017). China J Circ. 2017;32:34.

24. Chinese Diabetes Society. China guideline for type 2 diabetes (2017). Chinese J Diabetes. 2018;10.

25. China blood pressure measurement force group. The guideline of blood pressure measurement in China. Chin J Hypertens. 2015:23.

26. Chinese Nutrition Society:The Chinese dietary guideline 2016.

27. Isaka Y, Moriyama T, Kanda K. The SONG (salt intake and OrigiN from general foods) study - a large-scale survey of the eating habits and dietary salt intake in the working-age population. Intern Med. 2017;56:2423-30.

28. Li T, Qin Y, Lou P, Chang GQ, Chen PP, Qiao C, Zhang P, Zhang N. Salt intake and knowledge of salt intake in a Chinese population: a Crosssectional study. Intern Med. 2015;5

29. Hipgrave DB, Chang S, Li X, Wu Y. Salt and sodium intake in China. Jama. 2016;315:703-5.

30. Erdem Y, Arici M, Altun B, Turgan C, Sindel S, Erbay B, Derici U, Karatan O, Hasanoglu E, Caglar S. The relationship between hypertension and salt intake in Turkish population: SALTURK study. Blood Press. 2010;19:313-8.

31. Kumbla D, Dharmalingam M, Dalvi K, Ray S, Shah MK, Gupta S, Sadhanandham S, Ajmani AK, Nag S, Murthy S, et al. A study of salt and fat consumption pattern in regional Indian diet among hypertensive and dyslipidemic PaTients - SCRIPT study. J Assoc Physicians India. 2016; 64:47-54

32. Polonia J, Martins L, Pinto F, Nazare J. Prevalence, awareness, treatment and control of hypertension and salt intake in Portugal: changes over a decade. The PHYSA study. J Hypertens. 2014;32:1211-21.

33. Cogswell ME, Loria CM, Terry AL, Zhao L, Wang CY, Chen TC, Wright JD, Pfeiffer CM, Merritt R, Moy CS, Appel LJ. Estimated 24-hour urinary sodium and potassium excretion in US adults. Jama. 2018;319:1209-20.

34. Venezia A, Barba G, Russo O, Capasso C, De Luca V, Farinaro E, Cappuccio FP, Galletti F, Rossi G, Strazzullo P. Dietary sodium intake in a sample of adult male population in southern Italy: results of the Olivetti heart study. Eur J Clin Nutr. 2010:64:518-24.

35. Santos JA, Webster J, Land MA, Flood V, Chalmers J, Woodward M, Neal B, Petersen KS. Dietary salt intake in the Australian population. Public Health Nutr. 2017;20:1887-94 
36. Jensen PN, Bao TQ, Huong TTT, Heckbert SR, Fitzpatrick AL, LoGerfo JP, Ngoc TLV, Mokdad AH. The association of estimated salt intake with blood pressure in a Viet Nam national survey. PLoS One. 2018;13:e0191437.

37. Petermann-Rocha F, Sillars A, Brown R, Sweeney L, Troncoso C, GarciaHermoso A, Leiva AM, Martinez MA, Diaz-Martinez X, Poblete-Valderrama F, et al. Sociodemographic patterns of urine sodium excretion and its association with hypertension in Chile: a cross-sectional analysis. Public Health Nutr. 2019:1-10.

38. Bi Z, Liang X, XU A, Wang L, Shi X, Zhao W, Ma J, Guo X, Zhang X, Zhang J, et al. Hypertension prevalence, awareness, treatment, and control and sodium intake in Shandong Province, China: baseline results from Shandong-Ministry of Health action on salt reduction and hypertension (SMASH), 2011. Prev Chronic Dis. 2014;11:E88.

39. Stamler J, Chan Q, Daviglus ML, Dyer AR, Van Horn L, Garside DB, Miura K, Wu Y, Ueshima H, Zhao L, Elliott P. Relation of dietary sodium (salt) to blood pressure and its possible modulation by other dietary factors: the INTERMAP study. Hypertension. 2018;71:631-7.

40. Intersalt Cooperative Research Group. Intersalt: an international study of electrolyte excretion and blood pressure. Results for 24 hour urinary sodium and potassium excretion. Intersalt Cooperative Research Group. BMJ. 1988; 297:319-28.

41. Oh SW, Koo HS, Han KH, Han SY, Chin HJ. Associations of sodium intake with obesity, metabolic disorder, and albuminuria according to age. PLoS One. 2017;12:e0188770.

42. Schmidt RJ, Beierwaltes WH, Baylis C. Effects of aging and alterations in dietary sodium intake on total nitric oxide production. Am J Kidney Dis. 2001;37:900-8

43. Katsuya T, Ishikawa K, Sugimoto K, Rakugi H, Ogihara T. Salt sensitivity of Japanese from the viewpoint of gene polymorphism. Hypertens Res. 2003; 26:521-5.

44. He FJ, Marrero NM, MacGregor GA. Salt intake is related to soft drink consumption in children and adolescents: a link to obesity? Hypertension. 2008:51:629-34

45. Ma Y, He FJ, MacGregor GA. High salt intake: independent risk factor for obesity? Hypertension. 2015;66:843-9.

46. Zhang Y, Li F, Liu FQ, Chu C, Wang Y, Wang D, Guo TS, Wang JK, Guan GC, Ren KY, Mu J. Elevation of Fasting Ghrelin in Healthy Human Subjects Consuming a High-Salt Diet: A Novel Mechanism of Obesity? Nutrients. 2016;8.

47. Jin Y, Kuznetsova T, Maillard M, Richart T, Thijs L, Bochud M, Herregods MC, Burnier M, Fagard R, Staessen JA. Independent relations of left ventricular structure with the 24-hour urinary excretion of sodium and aldosterone. Hypertension. 2009;54:489-95.

48. Arora NK, Pillai R, Dasgupta R, Garg PR. Whole-of-society monitoring framework for sugar, salt, and fat consumption and noncommunicable diseases in India. Ann N Y Acad Sci. 2014:1331:157-73.

49. Sun Z, Zheng L, Detrano R, Zhang X, Xu C, Li J, Hu D, Sun Y. Risk of progression to hypertension in a rural Chinese women population with prehypertension and normal blood pressure. Am J Hypertens. 2010;23:627-32.

50. Jablonski KL, Racine ML, Geolfos CJ, Gates PE, Chonchol M, McQueen MB, Seals DR. Dietary sodium restriction reverses vascular endothelial dysfunction in middle-aged/older adults with moderately elevated systolic blood pressure. J Am Coll Cardiol. 2013;61:335-43.

51. Wang D, He Y, Li Y, Luan D, Yang X, Zhai F, Ma G. Dietary patterns and hypertension among Chinese adults: a nationally representative crosssectional study. BMC Public Health. 2011;11:925.

\section{Publisher's Note}

Springer Nature remains neutral with regard to jurisdictional claims in published maps and institutional affiliations.

Ready to submit your research? Choose BMC and benefit from:

- fast, convenient online submission

- thorough peer review by experienced researchers in your field

- rapid publication on acceptance

- support for research data, including large and complex data types

- gold Open Access which fosters wider collaboration and increased citations

- maximum visibility for your research: over $100 \mathrm{M}$ website views per year

At BMC, research is always in progress.

Learn more biomedcentral.com/submissions 